\title{
Atypical amplification of chromosome region 22q12 in melanoma: A case report
}

\author{
GIUSEPPINA LIGUORI ${ }^{1}$, MONICA CANTILE ${ }^{1}$, FRANCESCA COLLINA ${ }^{1}$, LAURA MARRA ${ }^{1}$, \\ ANNA DE CHIARA $^{1}$, RENATO FRANCO ${ }^{1}$, MICHELE CARAGLIA ${ }^{2}$, GERARDO BOTTI ${ }^{1}$, \\ MIRELLA D'ANDREA $^{3}$ and GIOVANNI FRANCESCO NICOLETTI ${ }^{3}$ \\ ${ }^{1}$ Pathology Unit, National Cancer Institute IRCCS 'Fondazione G. Pascale', Naples 80131; \\ Departments of ${ }^{2}$ Biochemistry, Biophysics and General Pathology, and ${ }^{3}$ Medical-Surgical and Dental Specialities, \\ Second University of Naples, Naples 80138, Italy
}

Received November 11, 2014; Accepted February 5, 2015

DOI: $10.3892 / 01.2015 .3150$

\begin{abstract}
Morphological, ultrastructural and immunohistochemical characteristics of clear cell sarcoma (CCS) of the soft tissue frequently overlap with those of malignant melanoma. Thus, the differential diagnosis between the two lesions represents an important diagnostic dilemma. However, a number of genetic factors can be used to differentiate the two tumors; in particular, the $\mathrm{t}(12 ; 22)(\mathrm{q} 13 ; \mathrm{q} 12)$ chromosomal translocation is typical of CCS, resulting in fusion of the EWSRI gene on chromosome 22q12 and the ATF1 gene on chromosome 12q13. The detection of this molecular alteration has proved useful in the differential diagnosis of the two lesions. The present study reports the case of a 71-year-old male patient with a suspicious lymph node mass. Immunohistochemical analysis of the lesion indicated a diagnosis of metastatic melanoma, however, cytogenetic analysis using fluorescence in situ hybridization was additionally performed to investigate the chromosomal rearrangements of the 22q12 region and completely exclude the possibility of CCS. The current case did not demonstrate the presence of the translocation, supporting the diagnosis of melanoma. However, a clear orange amplification signal
\end{abstract}

Correspondence to: Professor Michele Caraglia, Department of Biochemistry, Biophysics and General Pathology, Second University of Naples, 7 Via Luigi De Crecchio, Naples 80138, Italy

E-mail: michele.caraglia@unina2.it; michele.caraglia@alice.it

Mr. Gerardo Botti, Pathology Unit, National Cancer Institute IRCCS 'Fondazione G. Pascale', 52 Via Mariano Semmola, Naples 80131, Italy

E-mail: gbotti1@alice.it

Abbreviations: CCS, clear cell sarcoma; EWSR1, Ewing sarcoma breakpoint region 1; MHC, major histocompatibility complex; XBP, X box-binding protein; ER, endoplasmic reticulum; UPR, unfolded protein response

Key words: chromosomal translocation, clear cell sarcoma, differential diagnosis, EWSR 1 gene, fluorescence in situ hybridization was observed relative to an $\sim 500-\mathrm{kb}$ region adjacent to the EWSRI gene in the centromeric direction of chromosome $22 \mathrm{q} 12$. To the best of our knowledge, this is the first description of a $22 \mathrm{q} 12$ chromosomal alteration in melanoma. Furthermore, despite the presence of numerous genes in this region, their amplification has not previously been associated with the pathogenesis of melanoma.

\section{Introduction}

Melanoma is the most aggressive type of cutaneous malignancy and is responsible for $80 \%$ of mortalities from skin cancer, despite accounting for only $4 \%$ of all cases of dermatological cancer (1). Typically, melanoma is histologically diagnosed with the aid of specific protein markers, including S100, melanoma-associated antigen recognized by $\mathrm{T}$ cells-1 (MART-1) and human melanoma black-45 (HMB-45). Additionally, the diagnosis of malignant melanoma (MM) is commonly supported by the detection of $B R A F$ mutations (2).

By contrast, clear cell sarcoma (CCS), previously known as melanoma of the soft parts, is an uncommon type of malignant tumor. CCS typically presents as a deep soft-tissue mass, and microscopically and immunohistochemically demonstrates evidence of melanocytic differentiation (3).

While the majority of CCS occurs in the deep soft tissue, superficial variants have recently been reported in the dermis and/or superficial subcutis. Such CCS may be confused with primary melanoma or MM, as CCS and MM can stain positively for S100 and HMB-45 (4,5). Determination of the differential diagnosis of these tumors is facilitated by the result of a previously conducted cytogenetic study that identified the presence of a characteristic $\mathrm{t}(12 ; 22)$ chromosomal translocation involving the EWSRl gene in $70-90 \%$ of CCS cases (6).

The current study reports the case of a 71-year-old male who presented with a suspicious lymph node mass. The final diagnosis of melanoma was difficult despite the support of immunohistochemical analyses, thus, cytogenetic analysis was performed to detect chromosomal rearrangements of the $22 q 12$ region and exclude a diagnosis of CCS. Subsequently, a previously undescribed amplification of the chromosomal 

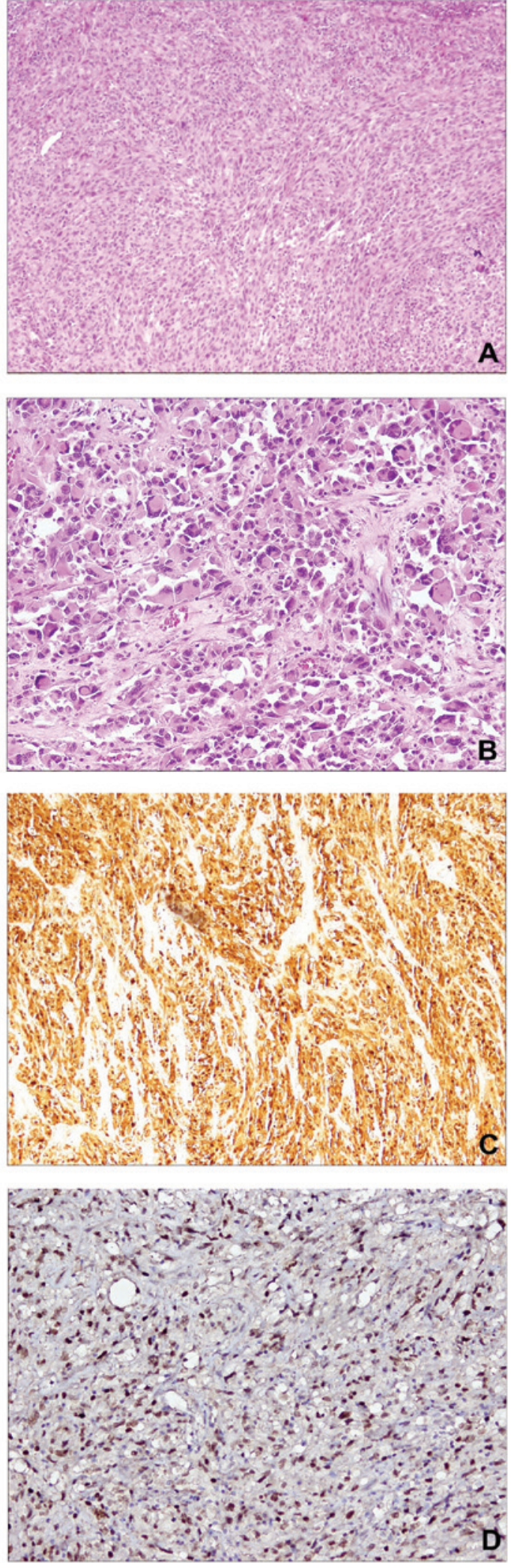

Figure 1. Lymph node sample analysis. Hematoxylin and eosin staining (A) representative of spindle cells and (B) epithelioid cells, and positive immunostaining for (C) S-100 and (D) MITF (magnification, x20).

area adjacent to the EWSRI gene in the centromeric direction was revealed. The study describes this chromosomal aberration and supports its presence by conducting molecular

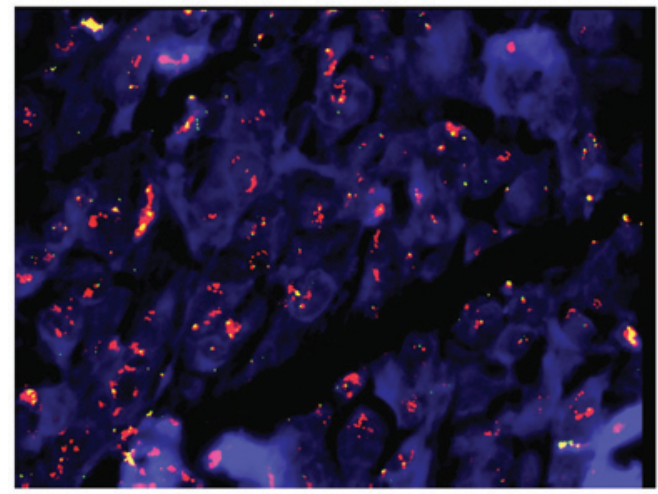

Figure 2. Application of the LSI Ewing sarcoma breakpoint region 1 Break Apart Rearrangement Probe in lymph node tissue revealed an increase in orange signals in the tumor cells, indicating an amplification signal with no gene rearrangement.

investigations into potential expression alterations of all genes included in the amplified area. Written informed consent was obtained from the patient.

\section{Case report}

A 71-year-old male was admitted to the Department of Melanoma and Soft Tissue Tumor Surgery of the National Cancer Institute IRCCS 'Fondazione G. Pascale' (Naples, Italy) due to the presence of a suspicious lymph node mass. An examination of the medical history revealed that the patient had underdone a surgical procedure six years previously to remove a skin tumor with a diagnosis of MM. Following surgical excision of the lymph node mass, macroscopic examination of the sample was used to characterize a whitish-colored mass measuring $8.0 \times 7.5 \times 6.5 \mathrm{~cm}$, with interspersed areas grossly consistent with necrosis. Focally, the lesion reached the margins of the exeresis (along the minor axis) and was surrounded by adipose tissue. Microscopically, the lesion was characterized by a hypercellular neoplasia with epithelioid and pleomorphic spindle cells, occasionally accompanied by the presence of abundant collagen (Fig. 1A and B). In addition to focal necrosis, evaluation of the mitotic activity index revealed 15 mitotic figures in 10 high-power fields (15/10 hpf), and residual lymph node parenchyma was identified on the mass boundary. Furthermore, immunohistochemical analysis identified strong positivity for S100 (polyclonal rabbit anti-human clone S100; cat. no. 760-2523; dilution, 1:100; Ventana Medical Systems, Inc., Tuscon, AZ, USA; Fig. 1C) and MITF (monoclonal mouse anti-human clone 34CA5; cat. no. NCL-MITF; dilution, 1:20; Leica Microsystems Ltd., Wetzlar, Germany; Fig. 1D) protein expression, and negativity for HMB-45 (monoclonal mouse anti-human clone gp100; cat. no. 790-4366; dilution, 1:100; Ventana Medical Systems, Inc.), MART-1 (monoclonal mouse anti-human A103; cat. no. ORG-8953; dilution, 1:50; Leica Microsystems Ltd.), muscle-specific actin (monoclonal mouse anti-human clone HHF35; cat. no. NCL-L-MSA; dilution, 1:500; Leica Microsystems Ltd.) and desmin (monoclonal mouse anti-human clone D33; cat. no. 760-2513; dilution, 1:50; Ventana Medical Systems, Inc.) markers.

To clarify the diagnosis, a molecular analysis was performed to assess the mutational status of the $B R A F$ gene. In brief, a Food and Drug Administration-approved 
Table I. Primers used for polymerase chain reaction analysis of the KREMEM1, ZNRF3 and XBP1 genes.

\begin{tabular}{lllrl}
\hline Gene & \multicolumn{1}{c}{ Forward primer (5'-3') } & \multicolumn{1}{c}{ Reverse primer (5'-3') } & $\begin{array}{c}\text { Product } \\
\text { length, bp }\end{array}$ & \multicolumn{1}{c}{$\begin{array}{c}\text { GenBank } \\
\text { reference number }\end{array}$} \\
\hline ZNRF3 & GGAGACCAGCAACCTCTCAC & CCATGCTTGTCCTCGTAGGG & 116 & NM_001206998.1 \\
KREMEN1 & GGGATGGAGTCAGGCTATGC & TGTTGCATTCGGTACTGGCT & 85 & NM_001039570.2 \\
XBP1 & AGCCAAGGGGAATGAAGTGAG & CTGCAGAGGTGCACGTAGTC & 76 & NM_005080.3 \\
\hline
\end{tabular}

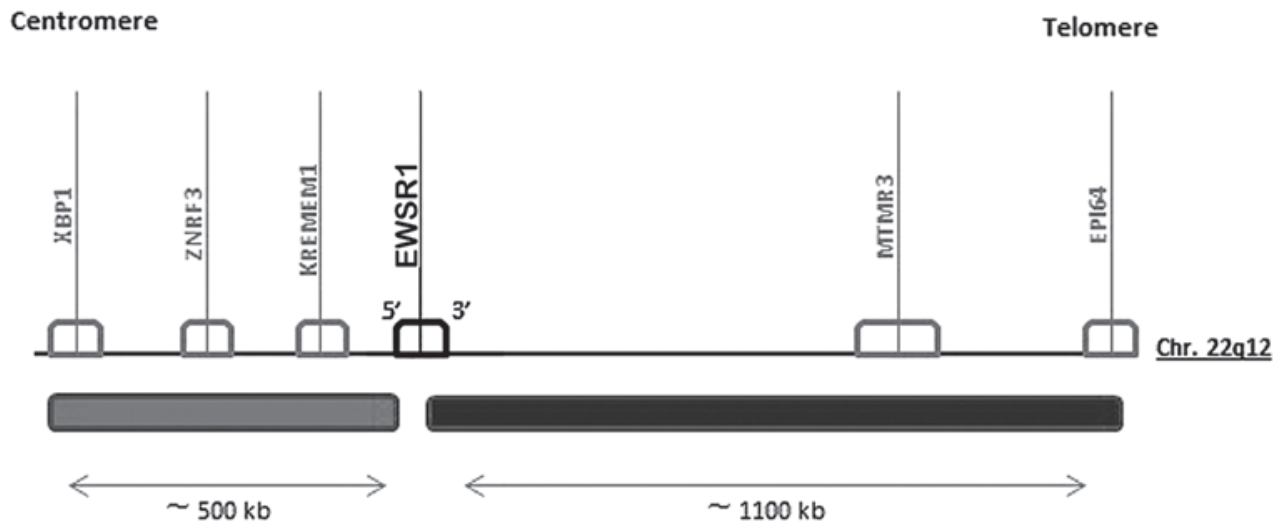

Figure 3. Schematic representation of chromosomal region 22q12, which was detected using the LSI Ewing sarcoma breakpoint region 1 Break Apart Probe. Genes to the left of the EWSR1 gene correspond to the orange signal in the centromeric direction.

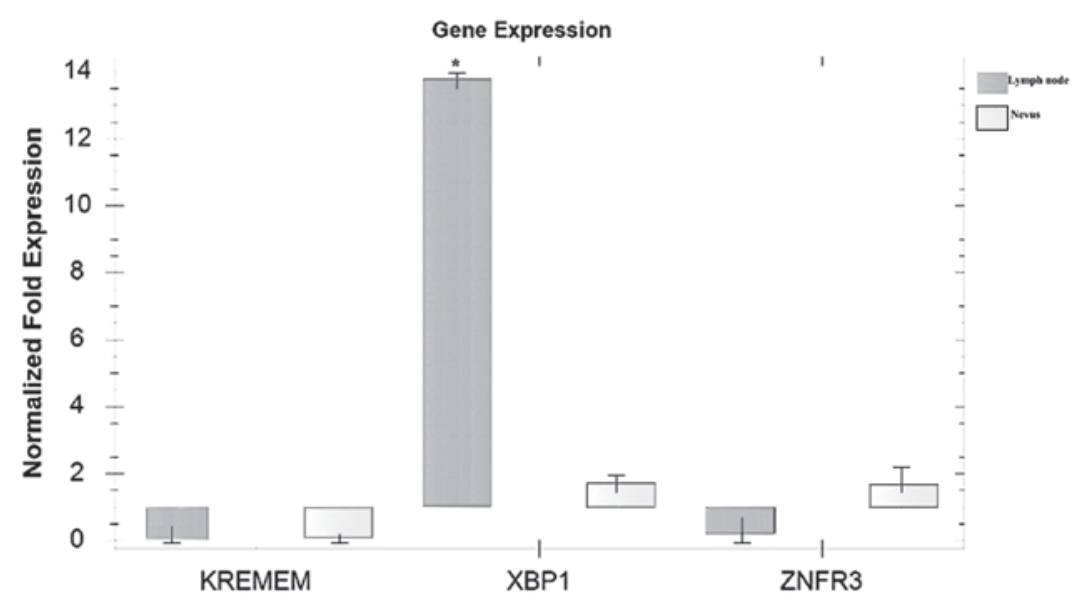

Figure 4. Qualitative polymerase chain reaction analysis of KREMEM1, ZNRF3 and XBP1 gene expression in metastatic lymph node and nevus samples. A significant difference in $X B P 1$ gene expression was identified in the lymph node and nevus samples $\left({ }^{*} \mathrm{P}<0.001\right)$. All experiments were performed in triplicate and data are expressed as the mean \pm standard deviation.

and CE-in vitro diagnostics-marked quantitative polymerase chain reaction (PCR)-based assay ( $\operatorname{cobas}^{\circledR} 4800 \mathrm{BRAF}$ V600 mutation test; Roche Molecular Systems, Inc., Branchburg, NJ, USA) was employed to identify potential mutations in codon 600, however, no mutations were identified in the $B R A F$ gene. Finally, to resolve the problem of a differential diagnosis with CCS, cytogenetic analysis was performed to investigate potential chromosomal rearrangements of the 22q12 region associated with the EWSRI gene translocation. In brief, the fluorescence in situ hybridization (FISH) method in conjunction with a Ewing sarcoma breakpoint region 1 (EWSR1) probe (Vysis LSI EWSR1 Dual Color Break Apart Probe; Abbott Molecular) was used to reveal a clear orange amplification signal relative to an $\sim 500-\mathrm{kb}$ region adjacent to the 5' EWSRI gene in the centromeric direction (Fig. 2).

Analysis of the amplified 22q12 chromosomal region (http://omim.org/geneMap/22/135?start=-3\&limit=10\&highlight=135) was performed, and the expression of only three genes adjacent to EWSR1, termed KREMEM1, ZNRF3 and XBPI, were identified (OMIM nos. 609898, 612062 and 194355, respectively; Fig. 3). 
To verify possible gene overexpression associated with chromosomal amplification, specific primers for each of the three genes were designed to enable the performance of gene expression analysis (Table I). Qualitative PCR analysis revealed marked elevation only in $X B P 1$ gene expression in the lymph node specimen compared with in the nevus sample, which was used as the non-neoplastic control (Fig. 4). However, variation in the gene or protein expression levels of $\mathrm{X}$ box-binding protein 1 (XBP1) may be observed in a larger series of melanoma samples, thus, a quantitative, large-scale study is required to clarify the results of the present study.

The clinical history of the patient, as well as the support of the in situ and molecular investigations, allowed a conclusive diagnosis of lymph node metastasis from melanoma with the presence of unconventional cytogenetic abnormalities to be determined in the present patient.

\section{Discussion}

The current study describes the case of a male patient with a previous history of melanoma, who presented with a suspicious lymph node mass. The clinical history, supported by morphological and immunohistochemical analysis, allowed a final diagnosis of lymph node metastasis from melanoma to be established, despite the analysis only demonstrating positivity for the S100 marker. For diagnostic and therapeutic purposes, the sample was additionally evaluated to determine the mutational state of the $B R A F$ gene, revealing an absence of nucleotide substitution.

Finally, considering the importance of the differential diagnosis between MM and CCS, particularly when the lesion occurs in the superficial side of the dermis, cytogenetic analysis was performed. Cytogenetic analysis was determined as essential for providing evidence of the potential presence of an EWSR 1 translocation with the ATF1 gene, which characterizes the majority of CCS cases.

FISH analysis identified the presence of gene translocations, however, an amplification of the $5^{\prime}$ region of the EWSRl gene in the centromeric direction was also identified. The presence of this chromosomal aberration has been sporadically described in Ewing's sarcoma (7), however, it has not previously been described in cutaneous melanoma, and a recent study reported aberrations of chromosome 22 in only a small percentage of acral melanomas (8). Thus, to the best of our knowledge, the current case represents the first description of the amplification of the $22 \mathrm{q} 12$ region in an MM patient. The chromosome region involved in the amplification and detected by the probe was $\sim 500 \mathrm{~kb}$ in length, and, in addition to EWSR1, includes three other genes: KREMEM1, ZNRF3 and XBP1.

KREMEM1, also termed KRM1, is a transmembrane receptor that functionally cooperates with Dickkopf-related protein 1 to block wingless-type MMTV integration site family (Wnt)/ $\beta$-catenin signaling $(9,10)$. The highest expression levels were previously described in heart, lung, kidney, skeletal muscle and neuroblastoma cell lines (10). Zinc and ring finger 3 (ZNRF3) is a zinc finger protein that exerts its activity as a cell-surface transmembrane E3 ubiquitin ligase. The protein acts as a negative regulator of the Wnt signaling pathway by mediating the ubiquitination and subsequent degradation of the Wnt receptor complex components Frizzled and low-density lipoprotein receptor-related protein 6. In particular, ZNRF3 appears to act as a tumor suppressor in colorectal tumor cell lines (11). XBP1, previously termed XBP2, is a transcription factor that regulates major histocompatibility complex (MHC) class II genes by binding to a promoter element referred to as $\mathrm{X}$ box. Furthermore, XBP1 is essential for hepatocyte growth, the differentiation of plasma cells and immunoglobulin secretion. Abnormal expression of MHC molecules in melanoma has been widely described in the literature (12), however, none of the three genes located in the $22 q 12$ chromosomal area have previously been directly associated with the pathogenesis of melanoma. Thus, possible alterations in the gene expression levels were evaluated in the present sample. The amplification of various chromosomal regions in tumors is often associated with the amplification/overexpression of specific genes $(13,14)$. In the present case, only one of the three genes, $X B P 1$, demonstrated abnormal expression levels compared with the non-neoplastic sample. Although the gene and protein expression values require quantitative confirmation in a larger series of melanoma cases, the data indicates specific functional characteristics of this marker. Notably, $X B P 1$ is activated during endoplasmic reticulum (ER) stress by unfolded protein response (UPR) and in particular, is activated by binding with inositol-requiring enzyme 1 , the most highly conserved signaling node of the UPR $(15,16)$. Cancer cells commonly undergo chronic ER stress, to which the cells have to adapt in order to survive and proliferate. In melanoma cells, intrinsic activation of the ER stress response is driven by oncogenic activation of mitogen-activated protein kinase kinase/extracellular signal-regulated kinase (17). Furthermore, a previous study observed that inhibition of $X B P 1$ expression decelerated melanoma cell proliferation and enhanced apoptosis induced by pharmacological ER stress inducers (17). Melanoma is the most frequent type of skin malignancy and is typically characterized by a poor prognosis associated with high metastatic capacity. Although numerous molecular pathways have been described for melanoma progression, the molecular mechanisms that result in metastatic development are not fully understood (1).

In conclusion, in the present study cytogenetic analysis revealed chromosomal rearrangement of chromosome $22 \mathrm{q} 12$, which was associated with the amplification of the XBPI gene. To the best of our knowledge, this is the first study to demonstrate an association between $22 \mathrm{q} 12$ chromosomal amplification and melanoma. Therefore, the identification of novel molecular markers may be useful for the improved diagnosis of melanoma, as well as for potentially indicating the prognostic and predictive value of various types of therapy.

\section{References}

1. Palmieri G, Capone M, Ascierto ML, et al: Main roads to melanoma. J Transl Med 7: 86, 2009.

2. Bhandaru M, Ardekani GS, Zhang G, et al: A combination of p300 and Braf expression in the diagnosis and prognosis of melanoma. BMC Cancer 14: 398, 2014.

3. Yang L, Chen Y, Cui T, Knösel T, Zhang Q, Geier C, Katenkamp D and Petersen I: Identification of biomarkers to distinguish clear cell sarcoma from malignant melanoma. Hum Pathol 43: 1463-1470, 2012. 
4. Kiuru M,Hameed M and Busam KJ: Compound clear cell sarcoma misdiagnosed as a Spitz nevus. J Cutan Pathol 40: 950-954, 2013.

5. Falconieri G, Bacchi CE and Luzar B: Cutaneous clear cell sarcoma: report of three cases of a potentially underestimated mimicker of spindle cell melanoma. Am J Dermatopathol 34: 619-625, 2012.

6. Song JS, Choi J, Kim JH, Jang SJ and Cho KJ: Diagnostic utility of EWS break-apart fluorescence in situ hybridization in distinguishing between non-cutaneous melanoma and clear cell sarcoma. Pathol Int 60: 608-613, 2010.

7. Szuhai K, IJszenga M, Tanke HJ, Taminiau AH, de Schepper A van Duinen SG, Rosenberg C and Hogendoorn PC: Detection and molecular cytogenetic characterization of a novel ring chromosome in a histological variant of Ewing sarcoma. Cancer Genet Cytogenet 172: 12-22, 2007.

8. Buckley PG, Mantripragada KK, Benetkiewicz M, et al: A full-coverage, high-resolution human chromosome 22 genomic microarray for clinical and research applications. Hum Mol Genet 11: 3221-3229, 2002.

9. Mao B, Wu W, Davidson G, et al: Kremen proteins are Dickkopf receptors that regulate Wnt/beta-catenin signalling. Nature 417: 664-667, 2002.

10. Nakamura T, Aoki S, Kitajima K, Takahashi T, Matsumoto K and Nakamura T: Molecular cloning and characterization of Kremen, a novel kringle-containing transmembrane protein. Biochim Biophys Acta 1518: 63-72, 2001.

11. Koo BK, Spit M, Jordens I, et al: Tumour suppressor RNF43 is a stem-cell E3 ligase that induces endocytosis of Wnt receptors. Nature 488: 665-669, 2012.
12. Degenhardt Y, Huang J, Greshock J, Horiates G, Nathanson K, Yang X, Herlyn M and Weber B: Distinct MHC gene expression patterns during progression of melanoma. Genes Chromosomes Cancer 49: 144-154, 2010.

13. Dei Tos AP, Doglioni C, Piccinin S, Sciot R, Furlanetto A, Boiocchi M, Dal Cin P, Maestro R, Fletcher CD and Tallini G: Coordinated expression and amplification of the MDM2, CDK4, and HMGI-C genes in atypical lipomatous tumours. J Pathol 190: 531-536, 2000.

14. Cantile M, Galletta F, Franco R, et al: Hyperexpression of $\mathrm{HOXC13}$, located in the $12 \mathrm{q} 13$ chromosomal region, in well-differentiated and dedifferentiated human liposarcomas. Oncol Rep 30: 2579-2586, 2013.

15. Hetz C, Martinon F, Rodriguez D and Glimcher LH: The unfolded protein response: integrating stress signals through the stress sensor IRE1 $\alpha$. Physiol Rev 91: 1219-1243, 2011.

16. He Y, Sun S, Sha H, et al: Emerging roles for XBP1, a sUPeR transcription factor. Gene Expr 15: 13-25, 2010.

17. Croft A, Tay KH, Boyd SC, et al: Oncogenic activation of MEK/ERK primes melanoma cells for adaptation to endoplasmic reticulum stress. J Invest Dermatol 134: 488-497, 2014. 